\title{
Performance Analysis of Impulse Denoising Techniques in Magnetic Resonance Imaging
}

\author{
Ram Paul Hathwal \\ ASET, Bijwasan, New Delhi
}

\author{
Rajesh Kumar Gupta \\ SoM, Thapar University, Patiala
}

\author{
Singara Singh Kasana \\ SoM, Thapar University, Patiala
}

\begin{abstract}
Medical images are corrupted by noise during their acquisition and transmission. Image denoising involves manipulation of the image data to produce a visually original quality image. The ultimate goal of medical image denoising technique is to compromise between the noise suppression and preservation of image details. The best possible information is required by the clinician for an accurate diagnosis. It has become an essential exercise especially in the Magnetic Resonance Imaging (MRI). In this work, we have taken magnetic resonance images infected with salt and pepper noise and have used three different de-noising techniques namely median filter, adaptive median filter, and a nonlinear cascade filter. All the three filters are used to reduce image noise at different densities and their Peak Signal to Noise Ratios (PSNR) are compared. This experimental analysis helps us increase the accuracy of MRI for easy diagnosis and determine which filter might be best suited for rectification of corrupted MRI.
\end{abstract}

\section{Keywords}

Medical Resonance Imaging, Median filter, Adaptive filter, Nonlinear cascade filter, PSNR.

\section{INTRODUCTION}

Magnetic resonance imaging (MRI) is one of the most widely used medical imaging tools in both clinical and research applications. The MRI acquired through medical resonance imaging equipments are often corrupted by noise during image acquisition, recording and transmission. Noise is any undesired information that contaminates the image. The presence of noise in an image may severely degrade the image quality and cause some loss of important information of the image. The source of contamination originates from the noisy channel transmission and errors induced during quantisation of the data [1].

Unfortunately, medical images encounter a various number of noises such as Gaussian, Poisson, Rician and impulse noise (salt and pepper noise) [4]. This performance analysis focuses on impulsive noise as medical resonance images are mainly prone to this type of noise. Salt and Pepper noise is an impulse type of noise and is also referred to as intensity spikes. This type of noise can be generally caused by dead pixels, analog-to-digital converter errors, bit errors in transmission, etc. It has only two possible values, a high value and a low value. The probability of each is typically less than 0.1 . The corrupted pixels are set alternatively to minimum or to maximum values giving the image "Salt and Pepper" like appearance [13]. The unaffected pixels remain Noise in MRI effects clinical visualisation for making diagnostic interpretations.

It is important to eliminate the noise contained in the images and at the same time preserving the image integrity. This purpose is best served by the use of image filters [1]. The objective of filtering an image is to remove specific impulses (noise) so that the noise free image is fully recovered with minimum image distortion. Image filters are used to attenuate or enhance particular frequencies easiest to visualize in the frequency domain. Image filtering is useful for many applications, including smoothing, sharpening, removing noise and edge detection.

Image denoising is done by filtering which can be broadly divided into categories: linear filtering and non-linear filtering [1]. Mean filtering and Gaussian filtering are the example of traditional spatial denoising methods. They are linear filters which cause blurring the images and simultaneously suppress the details. The performances of linear filters are not satisfactory because they blur the sharp edges; destroy lines and other finer details. To avoid these problems non-linear filtering is used which are popular in medical images filtering because of their mathematical simplicity. Although many methods have appeared in research literatures, each has its own advantages and limitations. The performance of a particular noise filter generally improves with the complexity of the implemented algorithm. On the other hand, methods with low complexity filter the salt-and-pepper noise at the expense of image details and textures. Thus it is a straight trade-off between complexity and efficiency.

In medical image processing, it is necessary to perform a high degree of noise reduction in an image before performing highlevel processing steps. Specifically for removal of salt-andpepper noise, conventional median filters and other classes of modified median filters [11], [6] are widely used. However, conventional median filtering would simply restore the processed pixel even when the pixel is a noise-free pixel but this may be avoided by modified median filters. The bestknown and most widely used non-linear digital filter is Standard Median Filter (SMF) which is based on order statistics [11]. These filters are known for their capability to remove impulse noise without damaging the edges. The main drawback of SMF is that it is effective only for low noise density. At high noise densities, SMF often results in loss of fine details of the image. They destroy the lines and sharp corners for large window sizes $(5 * 5$ or $7 * 7)$ and insufficient noise suppression for small window $(3 * 3)$ sizes [10], [5].

The drawback of SMF may be covered by proceeding adaptively [13]. This adaptive based median filter is known as Adaptive Median Filtering (AMF). In this filter, we can handle the local information of the image more exactly by taking a changing rectangular region in consideration. AMF can reduce the impulse noise with more accuracy and it seeks to preserve detail while smoothing non impulse noise, something that the SMF does not do.

The third filter under consideration is the Nonlinear Cascade Filter (NLCF). This method consists of two stages to enhance the filtering. The first stage is the Decision-based Median Filter (DMF) which is used to identify pixels likely to be 
contaminated by salt and pepper noise and replaces them by the median value. The second stage is the asymmetric trimmed filtering which is used to trim the noisy pixels in an asymmetric manner and processes with the remaining pixels. This can be achieved by Un-symmetric Trimmed Mean Filter (UTMF) or the Un-symmetric Trimmed Midpoint Filter (UTMP). The cascading of two stages provide the better denosing results, though the level of denoising in the first stage is lesser at high noise densities, the second stage helps to increase the noise suppression at higher frequency [9].

Hence, the presented cascaded filter, as a whole is very suitable for low, medium as well as high noise densities even above $90 \%$.

The remaining part of the paper is organized as follows. In Section II we briefly review the three algorithms and their structures. In Section III we review the experimental results analysis of the image with three samples of noise density for each filter. In Section IV, we provide comparison PSNR values of each filter in a table and draw a graph of noise density versus PSNR to show the comparison more clear. In Section V we give conclusions from the work and Section VI, we close with future scope.

\section{DENOISING ALGORITHMS}

This section covers the details regarding three non-linear algorithms of image denoising along with their theory.

\section{a). Standard Median Filter}

Linear filters are generally used to reduce noise in medical images. But this may lead to the blurring of edges and they do not reduce impulsive noise effectively from images. To overcome such a problem non-linear filters are used for noise reduction. These filters help to preserve edges and other fine details.

This filter may be represented by Eq (1).

$\mathrm{G}_{u, v}=$ median $\{I[x, y], \quad(x, y) \in w F\}$

where

$w F=\boldsymbol{w} x \boldsymbol{w}$ Filter window with pixel $(u, v)$ as its middle.

Standard Median Filter (SMF) is an example of non-linear filters. . SMF can remove the 'salt and pepper' noise from MRI image without disturbing the edges and other important features. The main idea of the SMF is to run through the MRI image entry by entry. In this technique, the ranking of the neighbouring pixels is done according to the intensity or brightness level of the pixels. A pattern of neighbouring pixels is chosen which is called the "window". For 1D signal, the most obvious window is just the first few preceding and following entries, whereas for 2D (or higher-dimensional) signals such as images, more complex window patterns are possible (such as "box" or "cross" patterns). Note that if the window has an odd number of entries, then the median is simple to define: it is just the middle value after all the entries in the window are sorted numerically. For an even number of entries, there is more than one possible median. Then the average of these medians is taken as a final median.

The functioning of the SMF can be explained step by step as following:

Step 1: Add a 2D medical image ( $\mathrm{M}$ by $\mathrm{N}$ ).

Step 2: Pre-allocate another matrix of size $(\mathrm{M}+2$ by $\mathrm{N}+2)$ with zeros.

Step 3: Copy the input matrix into the pre-allocated matrix.
Step 4: Form a window matrix of size (3 by 3 ) with the elements of input matrix.

Step 5: Copy the above window matrix into an array and sort it.

Step 6: Find the median element. Here $5^{\text {th }}$ element from ( 3 by 3) matrixes.

Step 7: Place the $5^{\text {th }}$ element into the output matrix. Do the procedure for the complete input matrixes.

Step 8: Convert the image into an Image of (0-255) gray scale type.

Step 9: Display the denoised image.

Here the number of pixels in the selected window is chosen odd which should be used to calculate the median [9]. Then a median is calculated by sorting all pixel values by their values, then selecting the median value as the new (denoised) value for the noisy pixel. After that, the value of pixel under evaluation is replaced by this new (denoised) value. Hence in SMF, we restored each and every pixel even when the pixel is a noise-free pixel. So the over smoothing of MRI image is occurred during this type of filtering when the noise density is high.

However, SMF would simply process noisy pixels as well as noise-free pixels, but it is the most widely used non-linear digital filters for MRI images. SMF is known for their capability to remove impulse noise without damaging the edges and other important features of MRI image. The main drawback of SMF is that it is effective only for low noise density. At high noise densities, SMF often results in loss of lines and sharp corners for large window of $(5 * 5$ or $7 * 7)$ sizes. It also results insufficient noise suppression for small window of $(3 * 3)$ size.

\section{b). Adaptive Median Filter}

The main purpose of adaptive median filter (AMF) is to reduce the 'Salt and Pepper' noise without the loss of fine details of MRI. It is used to smooth any non impulsive noise and to reduce excessive distortions such as too much thinning or thickening of the object boundaries of MRI image [9]. The working of this filter can be explained as follows:

The AMF works on a rectangular region $\mathbf{S}_{\mathbf{x y}}$. The AMF changes the size of $\mathbf{S}_{\mathbf{x y}}$ during the filtering operation depending on certain criteria as listed below. The output of the filter is a single value which the replaces the current pixel value at $(\mathrm{x}, \mathrm{y})$, the point on which $\mathbf{S}_{\mathbf{x y}}$ is centered at the time. The following notation is adapted from the book and is reintroduced here:

$\mathrm{Z}_{\min }=$ Minimum gray level value in $\mathrm{S}_{\mathrm{xy}}$

$\mathrm{Z}_{\max }=$ Maximum gray level value in $\mathrm{S}_{\mathrm{xy}}$

$\mathrm{Z}_{\text {med }}=$ Median of gray levels in $\mathrm{S}_{\mathrm{xy}}$

$\mathrm{Z}_{\mathrm{xy}}=$ gray level at coordinates $(\mathrm{x}, \mathrm{y})$

$\mathrm{S}_{\max }=$ Maximum allowed size of $\mathrm{S}_{\mathrm{xy}}$

This AMF works in two levels denoted by Level A and Level $\mathrm{B}$ as follows:

Level A:

[1] $\mathrm{A} 1=\mathrm{Z}_{\mathrm{med}}-\mathrm{Z}_{\mathrm{min}}$

[2] $\mathrm{A} 2=\mathrm{Z}_{\mathrm{med}}-\mathrm{Z}_{\max }$

[3] If A1 >0 AND A2 <0, Go to level B 
Else increase the window size

If window size $<=\mathrm{S}_{\max }$ repeat level $\mathrm{A}$

Else output $Z_{x y}$.

Level B:

[1] $\mathrm{B} 1=\mathrm{Z}_{\mathrm{xy}}-\mathrm{Z}_{\min }$

[2] $\mathrm{B} 2=\mathrm{Z}_{\mathrm{xy}}-\mathrm{Z}_{\min }$

[3] If $\mathrm{B} 1>0$ And $\mathrm{B} 2<0$ output $\mathrm{Z}_{\mathrm{xy}}$

Else output $\mathrm{Z}_{\text {med }}$.

\section{c). Non -linear Cascade Filter (NLCF)}

As we mentioned earlier, the non-linear cascade filtering is achieved in two stages to enhance the filtering and preserving the important features of medical images. The two stages are given below:

\section{i). Decision-based Median Filtering (DMF)}

Step 1: A 2-D window ' $S_{x y}$ ' of size $3 x 3$ is selected. Let $\mathrm{Z} x y$ be the processing pixel which lies in the center of the window.

Step 2: If $0<Z_{x y}<255$, then ' $Z_{x y}$ ' is considered to be a noise-free pixel and it is left unchanged.

Step 3: Otherwise, calculate the median of all the 9 pixels in the considered window and replace the processing pixel by the median value.

Step 4: Move the $3 \times 3$ window to the next pixel in the image.

The above steps are repeated for the entire image and the DMF output obtained is subjected to further processing. Fig. 1 shows the structure of DMF.

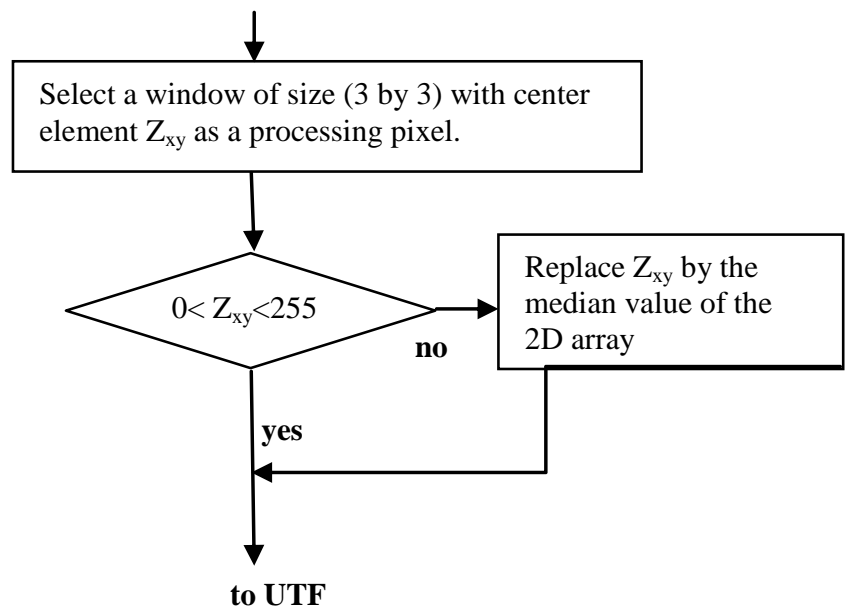

Fig 1 : Structure of DMF

\section{ii). Un-symmetric Trimmed Filter (UTF)}

The lower noise density is mostly removed by first stage DMF, but for higher density further suppression is needed. This can be achieved by trimming the extra noise by the asymmetric trimmed filtering. The un-symmetric trimmed filtering may be achieved in two ways based on the number of elements in a selected window.

\section{a). Un-symmetric Trimmed Mean Filtering (UTMF)}

Step 1: A 2-D window ' $S_{x y}$ ' of size $3 \times 3$ is selected.
Step 2: The pixel values in the window are stored in a 1-D array.

Step 3: If any pixel value in the array is either ' 0 ' or '255', the corresponding pixel values are eliminated from the array. Otherwise, all the 9 elements are retained.

Step 4: The pixel being processed is replaced by the mean of the values in the 1-D array.

We move the window by one step and repeat from step 1 to step 4 . The above steps are repeated, until the processing is completed for the entire image. Fig. 2 shows the structure of UTMF.

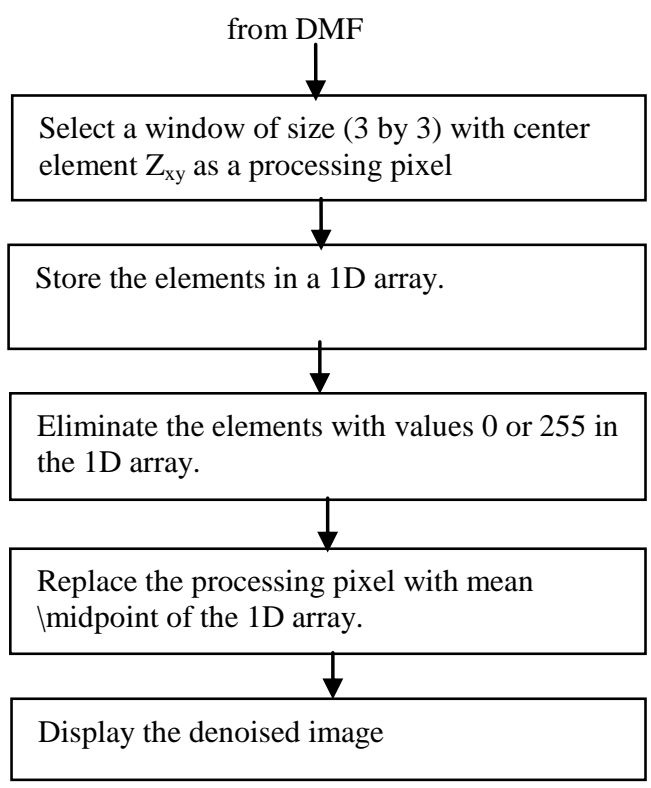

Fig 2: Structure of UTMF\UTMP

\section{b). Un-symmetric Trimmed Midpoint Filtering (UTMP)}

In UTMP, the impulses are trimmed unsymmetrically from both the ends, and midpoint is calculated from the maximum and minimum of the remaining values. The algorithm for UTMP is as follows:

Step 1: A 2-D window ' $S_{x y}$ ' of size $3 \times 3$ is selected.

Step 2: The pixel values in the window are sorted in ascending order, and stored in a 1-D array.

Step 3: If the pixel value in the array is either ' 0 ' or ' 255 ', the corresponding pixel values are eliminated, and the midpoint of trimmed or is calculated.

Step 4: The pixel being processed is replaced by the midpoint value calculated.

Step 5: If all the values are eliminated then the pixel is replaced by the processed neighboring pixel value of the array.

We move the window by one step, and repeat from step 2 to step 5. The above steps are repeated, until the processing is completed for entire image. 


\section{MEASUREMENT OF PERFORMANCE}

There is a trade off between the signal-to-noise ratio (SNR), spatial resolution and acquisition time required by clinical application in medical imaging. To measure the performance of the denoising techniques, several parameters are available for the comparison. Most common parameters are peak signal to noise ratio (PSNR), image quality index (IQI) and mean square error (MSE) etc [7].There are many other mathematical parameters using which the quality of denoised image can be calculated.

In this paper PSNR is chosen as the performance parameter. PSNR is the ratio between a signal's maximum power and the power of the signal's noise. Engineers commonly use the PSNR to measure the quality of reconstructed images that have been denoised. Image signals can have a wide dynamic range, so PSNR is usually expressed in decibels, which is a logarithmic scale. The PSNR is defined as:

$P S N R=10 \log _{10} \frac{M A X^{2}}{M S E} \mathrm{~dB}$

where $M A X$ is the maximum possible pixel value of the image. When the pixels are represented in gray scale using 8 bits per sample, which is equal to 255 . So MAX is equal to 255 X 255 and MSE is the mean square error between the denoised and original image [6]. It is calculated by taking the difference between two images pixel by pixel, square the result and finally average the results. The MSE for two $\mathrm{M} \times N$ images $I(i, j)$ and $\hat{I}(i, j)$ where one of the images is considered a noisy approximation of the other and is given by Eq (3):

$$
M S E=\frac{1}{M x N} \sum_{i=1}^{I} \sum_{j=1}^{J}[I(i, j)-\hat{I}(i, j)]^{2}
$$

The PSNR is most commonly used to measure quality of reconstruction of noisy image. A lower PSNR would normally indicate that the reconstruction is of lower quality and a higher PSNR would indicate that the reconstruction is of higher quality. One has to be extremely careful with the range of validity of this metric. Higher value of PSNR of denoised and original image implies that the performance of the denoising method is good. But it does not imply that the visual quality of the denoised image is good.

\section{EXPERIMENTAL ANALYSIS}

\section{A). Standard Median Filter}

i). Noise Density $=0.2$, Value of PSNR=22.1783

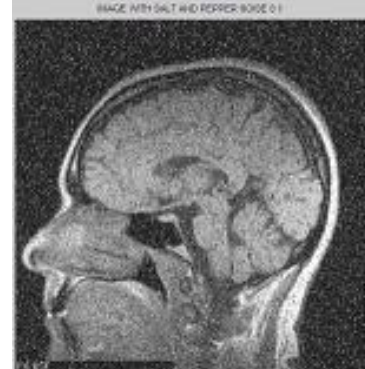

Noisy image

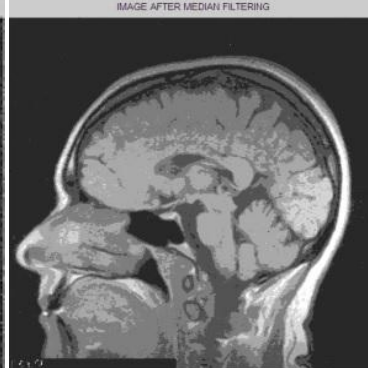

Denoised image i) Noise Density=0.4, Value of PSNR=17.3171

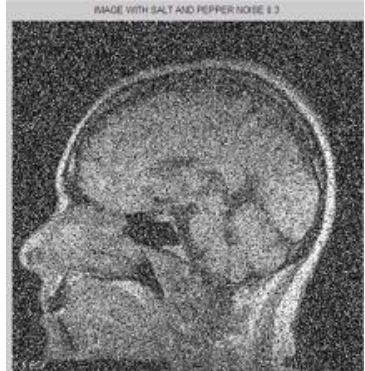

Noisy image

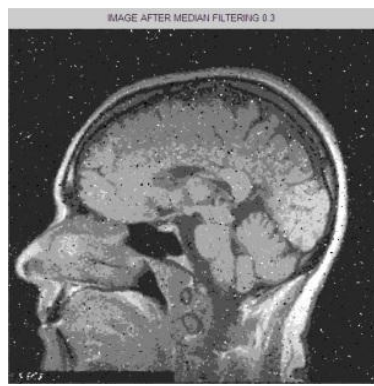

Denoised image iii). Noise Density $=0.7$, Value of PSNR $=9.5098$

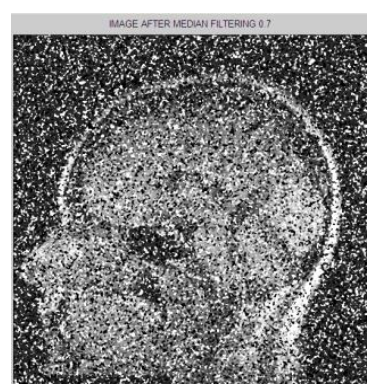

Noisy image

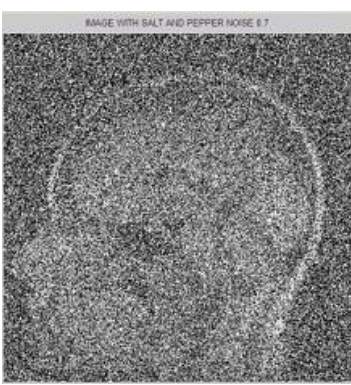

Denoised image

\section{B). Adaptive Median Filter}

i). Noise Density $=0.2$, Value of PSNR=23.7098

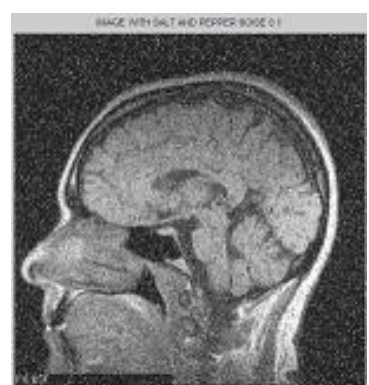

Noisy image

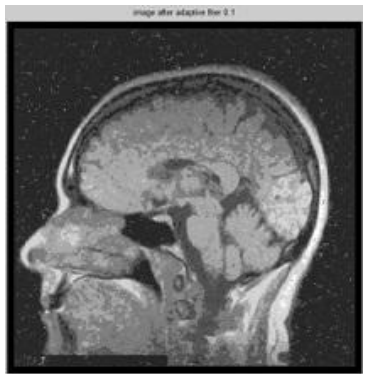

Denoised image ii). Noise Density $=0.4$, Value of PSNR $=23.2681$

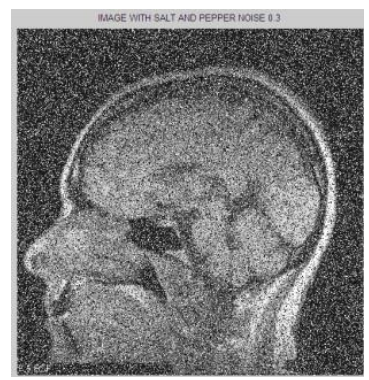

Noisy image

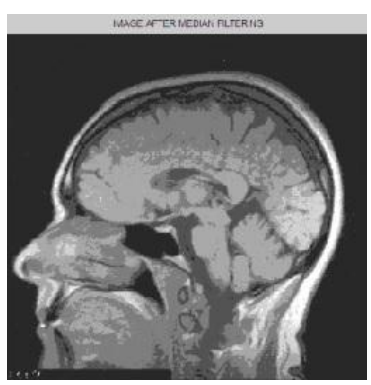

Denoised image iii). Noise Density $=0.7$, Value of PSNR $=21.0678$ 


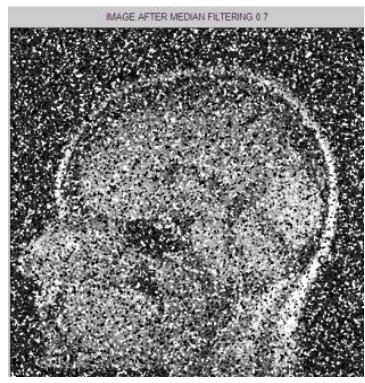

Noisy image

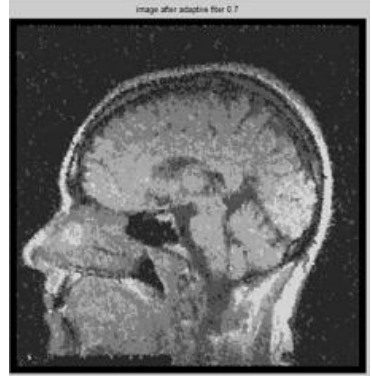

Denoised image

\section{C). Nonlinear Cascade Filter}

i). Noise Density=0.2, Value of PSNR=23.1393

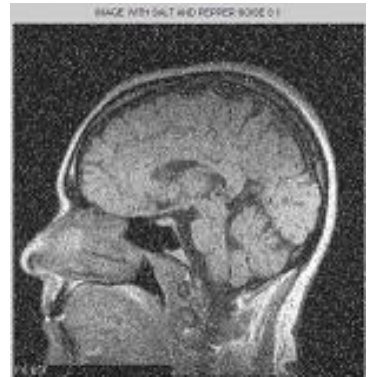

Noisy image

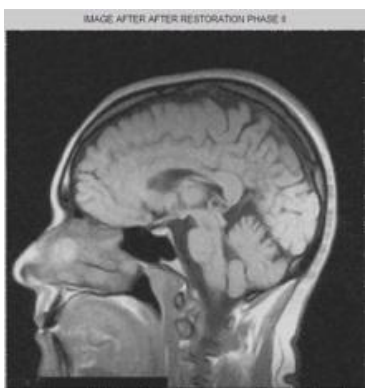

Denoised image ii). Noise Density $=0.4$, Value of PSNR $=22.5597$

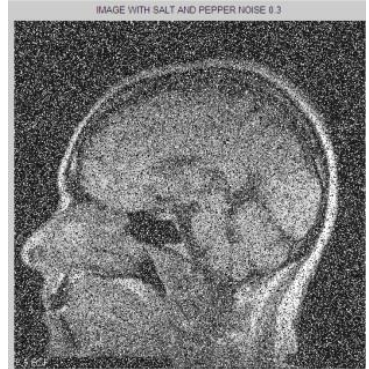

Noisy image

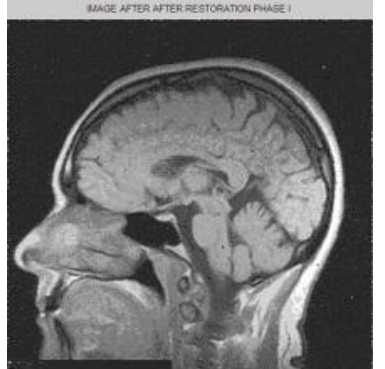

Denoised image iii). Noise Density $=0.7$, Value of PSNR=20.6791

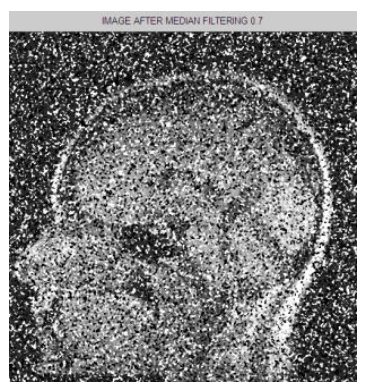

Noisy image

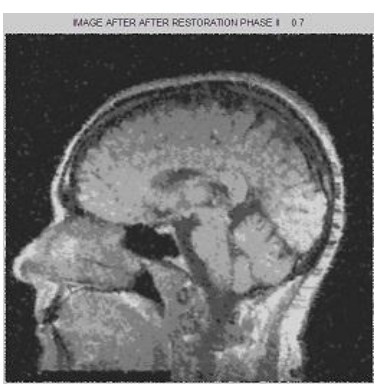

Denoised image

\section{COMPARATIVE RESULTS OF PSNR}

Peak signal to noise ratio (PSNR) between filtered images and original image is shown in table.I.These images are obtained by applying different type of median filtering technique with different values of variance from 0.1 to 0.9 as given below.

\section{a. Table I}

\begin{tabular}{|l|l|l|l|l|}
\hline $\begin{array}{l}\text { Noise } \\
\text { Density }\end{array}$ & $\begin{array}{l}\text { Noisy } \\
\text { Image }\end{array}$ & Median & AMF & NLCF \\
\hline 0.1 & 15.0895 & 22.899 & 23.8093 & 23.5274 \\
\hline 0.2 & 11.9791 & 22.1783 & 23.7098 & 23.1393 \\
\hline 0.3 & 10.274 & 20.2355 & 23.6984 & 23.1278 \\
\hline 0.4 & 9.0296 & 17.3171 & 23.2681 & 22.5597 \\
\hline 0.5 & 8.0503 & 14.1794 & 22.6498 & 21.9415 \\
\hline 0.6 & 7.2556 & 11.7926 & 21.8609 & 21.4090 \\
\hline 0.7 & 6.586 & 9.5098 & 21.0678 & 20.6791 \\
\hline 0.8 & 6.0101 & 8.6968 & 19.7218 & 19.6665 \\
\hline 0.9 & 5.5002 & 8.0518 & 17.6627 & 18.0366 \\
\hline
\end{tabular}

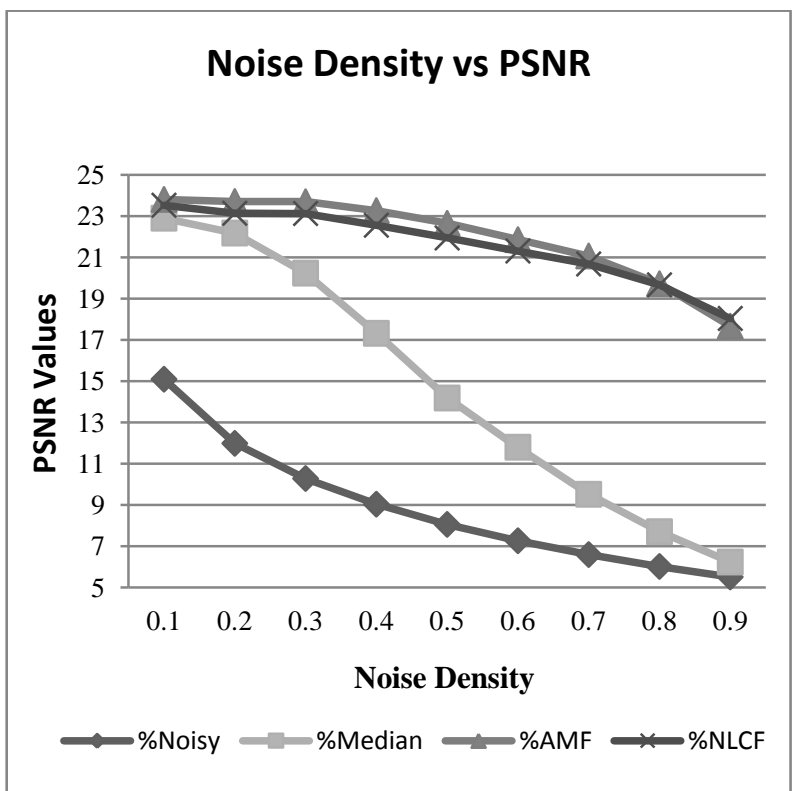

\section{b. Graph}

\section{CONCLUSION}

In this paper, we demonstrated the MRI denoising results obtained from three denoising algorithms namely Standard Median Filter (SMF), Adaptive Median Filter (AMF) and Nonlinear Cascade Filter (NLCF). We considered all the range of noise densities for a MRI image. To analyze the noise suppression and edge preservation capability of the proposed approach, different performance indices have been used. The MSE and PSNR are the parameters to measure the noise suppression capability. The presented results demonstrate the usefulness of image denoising for visual enhancement of MRI image as well as for improving some further automatic processing like the segmentation of MRI images.

In case of the low noise density, all these nonlinear filters are effective approximately but AMF and NLCF perform better than SMF in terms of the Peak Signal-to-Noise Ratio (i.e. PSNR). 
In case of the medium noise density, the performance of SMF is not satisfactory. It is evident that the NLCF and AMF perform better than SMF in terms of in terms of PSNR and visual perception.

In case of the higher noise density, the NLCF performs best and it is due to the cascading of two noise density based schemes in NLCF. For instance at noise variance of 0.7 the PSNR of the restored image improves by about $14 \mathrm{db}$ as compared to the noisy image as opposed to the case of $11 \mathrm{db}$ for AMF. AMF perform better than SMF in terms of PSNR and visual perception. The results of SMF are worst at higher noise densities. By investigating the comparison parameters, the NLFC, as a whole is very suitable for low, medium as well as high noise densities.

Visual study of the processed image by these methods shows that impulses are reduced at the cost of appearance of the image textures and other fine details. Apart from the visualization, the performance has been extensively analyzed and evaluated based on the obtained values of the performance indices (PSNR) as mentioned above.

\section{FUTURE SCOPE}

In the experimental analysis given above we have tried to determine the best method out of the given three filters. All the three filters are non linear. Therefore, the future work can be done that includes more non linear filters as more and more work on new techniques is being done in this field. Also the conventional linear filters can be included in the study. A totally new direction could possibly include the upcoming field of wavelet based filters. Similar bodies of research work may be conducted for other kinds of non-grayscale medical images.

\section{REFERENCES}

[1] S. J. Ko and Y. H. Lee, "Center weighted median filters and their applications to image enhancement", IEEE Transactions on Circuits and Systems, vol. 38, no. 9, pp. 984-993, 1991

[2] T. Sun and Y. Neuvo, "Detail-preserving median based filters in image processing", Pattern Recognition Letters, vol. 15, no. 4, pp. 341-347, 1994.

[3] B. Jeong and Y. H. Lee, "Design of weighted order statistic filters using the perceptron algorithm," IEEE Transaction Signal Processing, vol. 42, no. 11, pp. 3264-3269, 1994.

[4] A. Macovski, "Noise in MRI", Magn. Reson. Medical, vol. 36, pp. 494-497, 1996.

[5] E. Abreu, M. Lightstone, S. K. Mitra, and K. Arakawa, "A new efficient approach for the removal of impulse noise from highly corrupted images", IEEE Transactions Image Processing, vol. 5, no. 6, pp. 1012-1025, 1996.

[6] T. Chen, K. K. Ma, and L. H. Chen, "Tri-state median filter for image denoising", IEEE Transactions on Image Processing, vol. 8, no. 12, pp. 1834-1838, 1999.

[7] A. Achim, A. Bezerianos, and P. Tsakalides, "Novel Bayesian multiscale method for noise removal in medical MRI images," IEEE Transactions Medical Imaging, vol. 20, no. 8, pp. 772-783, 2001.

[8] H.-L. Eng and K.-K. Ma, "Noise adaptive soft-switching median filter," IEEE Transactions on Image Processing, vol. 10, pp. 242-251, 2001.

[9] A. Samsonov and C. Johnson "Noise-Adaptive Nonlinear Diffusion Filtering of MR Images With Spatially Varying Noise Levels", Magnetic Resonance in Medicine, vol. 52, pp. 798-806, 2004.

[10] R. H. Chan, C. W. Ho, and M. Nikolova, "Salt-andPepper Noise Removal by Median-Type Noise Detectors and Detail-Preserving Regularization," IEEE Transaction on Image Process., vol. 14, no. 10, pp. 1479-1485, 2005.

[11] A. Buades, B. Coll, and J. M. Morel, "A Review of Image Denoising Algorithms, with a new one", Journal of Multiscale Modeling and Simulation, vol.4, no.2, pp. 490-530, 2005.

[12] W. Luo, "An efficient detail-preserving approach for removing impulse noise in images", IEEE Sign al Process. Lett., vol. 13, no. 7, pp.413-416, 2006.

[13] T. C. Lin, P. T. Yu, "A new adaptive center weighted median filter for suppressing impulsive noise in images", Information Sciences 177, pp. 1073-1087, 2007.

[14] D. Yiqiu, and S. Xu, "A New Directional Weighted Median Filter for Removal of Random-Valued Impulse Noise", IEEE Signal Processing Letters, vol. 14, no. 3,pp. 193-196, 2007.

[15] S. Balasubramanian, S. Kalishwaran, R. Muthuraj, D. Ebenezer, V. Jayaraj , " An Efficient Non-linear Cascade Filtering Algorithm for Removal of High Density Salt and Pepper Noise in Image and Video sequence". International conference on "Control, Automation, Communication and Energy Conservation 2009", 2009.

[16] L. Mitiche, "A review of wavelet denoising in MRI and ultrasound imaging," Recent Advances in Intelligent Computational Systems(RAICS), vol. 20, no. 8, pp. $772-$ 783, 2011. 